\title{
Antiferromagnetic phase transition and spin correlations in $\mathrm{NiO}$
}

\author{
Chatterji, Tapan; McIntyre, G.J.; Lindgård, Per-Anker
}

Published in:

Physical Review B. Condensed Matter and Materials Physics

Link to article, DOI:

10.1103/PhysRevB.79.172403

Publication date:

2009

Document Version

Publisher's PDF, also known as Version of record

Link back to DTU Orbit

Citation (APA):

Chatterji, T., Mclntyre, G. J., \& Lindgård, P-A. (2009). Antiferromagnetic phase transition and spin correlations in NiO. Physical Review B. Condensed Matter and Materials Physics, 79(17), 172403.

https://doi.org/10.1103/PhysRevB.79.172403

\section{General rights}

Copyright and moral rights for the publications made accessible in the public portal are retained by the authors and/or other copyright owners and it is a condition of accessing publications that users recognise and abide by the legal requirements associated with these rights.

- Users may download and print one copy of any publication from the public portal for the purpose of private study or research.

- You may not further distribute the material or use it for any profit-making activity or commercial gain

- You may freely distribute the URL identifying the publication in the public portal

If you believe that this document breaches copyright please contact us providing details, and we will remove access to the work immediately and investigate your claim. 


\title{
Antiferromagnetic phase transition and spin correlations in $\mathrm{NiO}$
}

\author{
Tapan Chatterji, ${ }^{1}$ G. J. McIntyre, ${ }^{2}$ and P.-A. Lindgard ${ }^{3}$ \\ ${ }^{1}$ JCNS, Forschungszentrum Jülich, Outstation at Institut Laue-Langevin, B.P. 156, 38042 Grenoble Cedex, France \\ ${ }^{2}$ Institut Laue-Langevin, B.P. 156, 38042 Grenoble Cedex, France \\ ${ }^{3}$ Riso National Laboratory, DK-4000 Roskilde, Denmark \\ (Received 15 December 2008; published 6 May 2009)
}

\begin{abstract}
We have investigated the antiferromagnetic (AF) phase transition and spin correlations in NiO by hightemperature neutron diffraction below and above $T_{N}$. We show that $\mathrm{AF}$ phase transition is a continuous second-order transition within our experimental resolution. The spin correlations manifested by the strong diffuse magnetic scattering persist well above $T_{N} \approx 530 \mathrm{~K}$ and could still be observed at $T=800 \mathrm{~K}$ which is about $1.5 T_{N}$. We argue that the strong spin correlations above $T_{N}$ are due to the topological frustration of the spins on a fcc lattice. The Néel temperature is substantially reduced by this process. We determined the critical exponents $\beta=0.328 \pm 0.002$ and $\nu=0.64 \pm 0.03$ and the Néel temperature $T_{N}=530 \pm 1 \mathrm{~K}$. These critical exponents suggest that $\mathrm{NiO}$ should be regarded as a $3 d X Y$ system.
\end{abstract}

DOI: 10.1103/PhysRevB.79.172403

Transition-metal oxides have been the subject of renewed interest ever since high-temperature superconductivity was discovered in cuprate materials. The transition-metal oxides with narrow $d$ bands form strongly correlated electron systems or a Mott-Hubbard system in which Coulomb interactions between electrons lead to a breakdown of the conventional band theory. In such a system, hole or electron doping may transform an insulating antiferromagnet into a highly correlated metal. ${ }^{1,2}$ In selected copper oxides, the metal can superconduct, while in others it is a strongly renormalized Fermi liquid. In manganites for example the doping transforms the insulating antiferromagnet into a ferromagnetic metal with colossal magnetoresistive properties. ${ }^{3}$

Among the transition-metal oxides the antiferromagnetic $\mathrm{NiO}$ plays a key role in the studies of the electronic and magnetic properties of correlated $3 d$-electron system. NiO, along with other transition-metal oxides $\mathrm{MnO}, \mathrm{FeO}$, and $\mathrm{CoO}$, crystallizes with the face-centered-cubic (space group $F m \overline{3} m$ ) NaCl-type structure. The magnetic properties of pure $\mathrm{NiO}$ are well known. $\mathrm{Ni}^{2+}$ ions in $\mathrm{NiO}$ are in an $S=1$ state and order at $T_{N}=530 \mathrm{~K}$ in the type-II antiferromagnetic structure with the propagation vector $\mathbf{k}=\left(\frac{1}{2}, \frac{1}{2}, \frac{1}{2}\right)$. Ferromagnetic (111) layers are antiferromagnetically stacked along the [111] direction. The magnetic moments of Ni lie in the (111) plane and there is now considerable evidence that they lie in the $[1,1,-2]$ direction. ${ }^{4}$ There is a rhombohedral distortion at $T_{N}$ which increases as the temperature is lowered. The electronic structure of $\mathrm{NiO}$ has been extensively investigated. $^{5-7}$ There are strong indications that $\mathrm{NiO}$ is a charge-transfer insulator ${ }^{5}$ in which the top of the valence band is primarily formed by oxygen $2 p$ states, while the bottom of the conduction band is Ni-3d like. The insulating state is characterized by a band gap of about $4 \mathrm{eV}$. Magnetic exchange interactions in $\mathrm{NiO}$ have been calculated ${ }^{7-9}$ and have also been determined by measuring the spin-wave dispersion by inelastic neutron scattering. ${ }^{10}$ The spin-wave dispersion in $\mathrm{NiO}$ could be described by a nearest-neighbor $(\mathrm{NN})$ ferromagnetic exchange interaction $J_{1}=1.37 \mathrm{meV}$ and a next-nearest-neighbor (NNN) antiferromagnetic exchange interaction $J_{2}=-19.01 \mathrm{meV}$. In addition an anisotropy of the form $D_{1} S_{x}^{2}+D_{2} S_{y}^{2}$ where $x$ is perpendicular to the plane, and
PACS number(s): $75.25 .+\mathrm{z}$

$y$ within, is needed to describe the data, with $D_{1}=1.13 \mathrm{~K}$ and $D_{2}=0.06 \mathrm{~K}$. These parameters give a mean-field Néel temperature $^{10}$ of $T_{N}(\mathrm{MF})=886 \mathrm{~K}$. The calculated exchange interaction values are very close to these experimental values. In a recent paper Kotani and Schilfgaarde ${ }^{11}$ calculated the spin-wave dispersion for $\mathrm{NiO}, \mathrm{MnO}$, and $\alpha$-MnAs based on quasiparticle self-consistent GW (QSGW) method and by local-density approximation. They obtained $J_{1}^{+}=0.77 \mathrm{meV}$, $J_{1}^{-}=1.00 \mathrm{meV}$, and $J_{2}=-14.7 \mathrm{meV}$. However the calculated value of $T_{N}=275 \mathrm{~K}$ is much lower than the experimental value of $T_{N} \approx 530 \mathrm{~K}$. The $\mathrm{NN}$ and $\mathrm{NNN} \mathrm{Ni}^{2+}$ ions in $\mathrm{NiO}$ are linked by $90^{\circ}$ and $180^{\circ}$ superexchange paths involving one intervening $\mathrm{O}^{-2}$ anion. The strong NNN exchange interaction is due to the $180^{\circ} \mathrm{Ni}-\mathrm{O}-\mathrm{Ni}$ superexchange interaction caused by the overlap of the $\mathrm{Ni}^{2+}\left(3 d^{8}\right) e_{g}$ orbitals with the $\mathrm{O}^{2-} p_{\sigma}$ orbitals. $J_{2}$ was first derived by Anderson ${ }^{12}$ as $J_{2}=(10 D q / 3)^{2} / U_{\text {eff }}$, where $10 D q$ is the crystal-field splitting and $U_{\text {eff }}$ is the Coulomb interaction. The crystal-field splitting $10 D q$ has been determined ${ }^{13}$ by resonant inelastic x-ray scattering to be $1.05 \mathrm{eV}$ and first-principles calculations ${ }^{9}$ gave $U_{\text {eff }}=6.3 \mathrm{eV}$. Using these values $J_{2}$ is calculated to be $J_{2}=19.44 \mathrm{meV}$ which is surprisingly close to the $J_{2}=19.01 \mathrm{meV}$ determined from inelastic neutron scattering. ${ }^{10}$ The weak ferromagnetic $\mathrm{NN}$ interaction is presumably due to the combined effects of superexchange and direct exchange. The first-principles calculation ${ }^{9}$ of $J_{1}$ is also very close to the experimental value. ${ }^{10}$ So in $\mathrm{NiO}$ the NNN superexchange interaction $J_{2}$ is much stronger than the direct $\mathrm{NN}$ interaction $J_{1} . J_{2}$ determines the magnetic ordering and related properties whereas the weak $J_{1}$ has been proved to play a decisive role in the weak structural distortion. ${ }^{14-16}$ In fact due to the small rhombohedral distortion below $T_{N}$, there are two different values of $J_{1}$ denoted by $J_{1}^{+}$and $J_{1}^{-}$, where the plus sign denotes coupling between neighbors having antiparallel spin orientation and the minus sign denotes that between neighbors having parallel spin orientations. Such inequivalence between NNN neighbors can perhaps be ignored, however.

$\mathrm{Ni}^{2+}$ ions are situated on a fcc lattice which is frustrated with regard to the nearest-neighbor Heisenberg interaction. Such frustrated antiferromagnets usually have a wealth of different ordered magnetic phases and often show short- 
range order or antiferromagnetic correlations well above the Néel temperature. The magnetic diffuse neutron-scattering cross section is directly proportional to the generalized wave-vector-dependent susceptibility tensor and thus measures the Fourier components of the microscopic magnetization fluctuations. ${ }^{17}$ Such measurements have been reported in various magnetic systems ${ }^{18-22}$ and the magnetic exchange interactions have been determined from the diffuse magnetic neutron-scattering cross sections. Although $\mathrm{NiO}$ is a prototype of a strongly correlated transition-metal oxide with a type-II antiferromagnetic structure, diffuse magnetic scattering investigation of $\mathrm{NiO}$ above the ordering temperature has not been reported so far. We report here the results of investigation of the spin correlations in $\mathrm{NiO}$ above the Néel temperature by high-temperature neutron diffraction. We also investigated the antiferromagnetic phase transition at $T_{N} \approx 530 \mathrm{~K}$ with a very good temperature resolution. The motivation behind this study was to investigate the nature of the phase transition that has remained controversial. In the past it has been debated whether the antiferromagnetic phase transition in $\mathrm{NiO}$ has a weak first-order character close to $T_{N}$. In fact renormalization group theory ${ }^{25}$ and Monte Carlo simulations $^{26-29}$ suggest a first-order transition. However the neutron-diffraction investigations of van Doorn and Du Plessis $^{30}$ suggested a continuous phase transition within their experimental resolution. Our present neutron-diffraction investigation with a much better temperature resolution shows that the AF phase transition in $\mathrm{NiO}$ is indeed continuous.

A large single crystal of $\mathrm{NiO}$ of size of $15 \times 15 \times 25 \mathrm{~mm}^{3}$ was mounted inside a high-temperature furnace with its $[1,-1,0]$ crystallographic axis approximately parallel to the $\omega$ axis of the diffractometer D10 at the Institut Laue-Langevin in Grenoble. A vertically focusing pyrolytic graphite $\mathrm{PG}(002)$ monochromator gave a neutron wavelength of $\lambda=2.36 \AA$. To suppress the higher-order wavelength contamination we used a PG(002) filter. To reduce the background for some scans for the diffuse scattering we used a PG(002) analyzer. The lattice constant of the crystal was determined by centering seven nuclear reflections and was found to be $a=4.182 \pm 0.001 \AA$ at $T=292 \mathrm{~K}$.

We investigated the antiferromagnetic phase transition of $\mathrm{NiO}$ close to $T_{N}$ by measuring the temperature variation of the $\frac{1}{2} \frac{1}{2} \frac{1}{2}$ magnetic reflection without the analyzer. The results are shown in Fig. 1 (top). The intensity of this reflection decreases continuously with increasing temperature and becomes zero at about $T_{N} \approx 530 \mathrm{~K}$. Our present experimental results show that the intensity of the $\frac{1}{2} \frac{1}{2} \frac{1}{2}$ magnetic reflection of $\mathrm{NiO}$ varies continuously within our experimental resolution, which is better than in previous measurements. ${ }^{30}$ The data just below $T_{N}$ could be fitted by a power-law exponent

$$
I=I_{0}\left(\frac{T_{N}-T}{T_{N}}\right)^{2 \beta},
$$

where $I$ is the integrated intensity, $I_{0}$ is the saturation value of the intensity at $T=0, T_{N}$ is the critical temperature, and $\beta$ is the power-law exponent. Least-squares fit of the data in the temperature range from $T=515$ to $T=528 \mathrm{~K}$ gave $\beta=0.328 \pm 0.002$ and a Néel temperature $T_{N}$
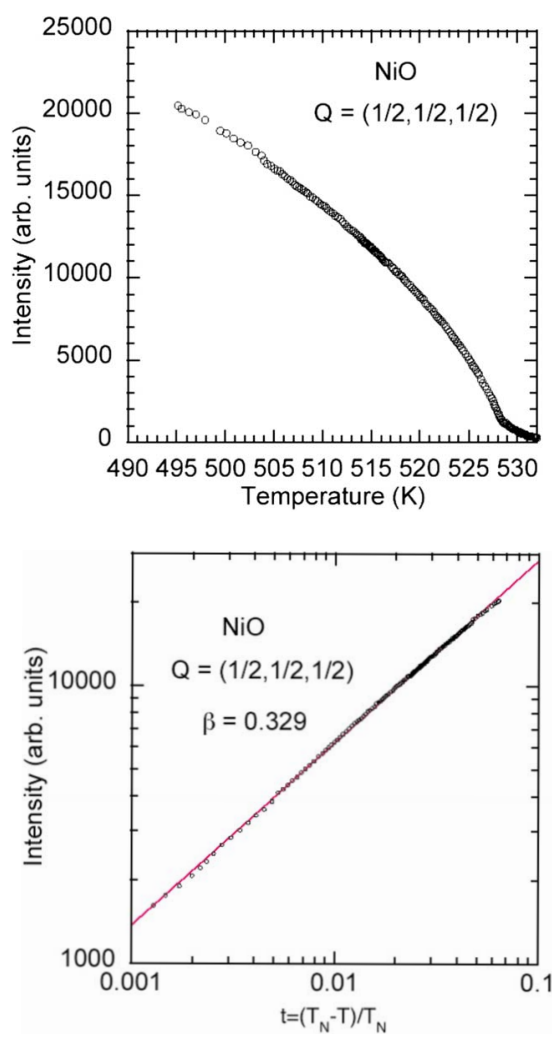

FIG. 1. (Color online) (a) Temperature dependence of the $\frac{1}{2} \frac{1}{2} \frac{1}{2}$ magnetic reflection of $\mathrm{NiO}$. (b) Log-log plot of the intensity as a function of the reduced temperature $t=\left(T_{N}-T\right) / T_{N}$. From the slop of the linear plot the critical exponent is determined to be $\beta$ $=0.329 \pm 0.002$.

$=528.84 \pm 0.02 \mathrm{~K}$. We believe that this procedure gave the correct value of the Néel temperature and that there was no appreciable correlation between the fit parameters. We started the refinements with very different initial parameters but always ended up with the same results for $T_{N}$ and the corresponding critical exponent $\beta$ showing that the correlation between them was negligible. We also checked the correlation matrices of the least-squares fits and found them to be not strongly correlated. Also the Néel temperature obtained in this way agreed well with the value obtained from the power-law fit of the temperature variation in the inverse correlation length to be described later. The fitted value of the Néel temperature was used to determine the reduced temperature $t=\left(T_{N}-T\right) / T_{N}$. We then produced a standard log-log plot to extract the critical exponent $\beta=0.329$ by a linear regression that agreed well with that determined by the leastsquares fit.

We made $Q$ scans through $Q=\left(\frac{1}{2} \frac{1}{2} \frac{1}{2}\right)$ along [111] and perpendicular to this direction at temperatures below and above $T_{N}$ from the same NiO single crystal but in a different experiment. Figure 2 shows a few such scans parallel to [111]. At $T=519.6 \mathrm{~K}$ a resolution-limited sharp Gaussian peak was observed. The peak became broader at temperatures above $T_{N} \approx 530 \mathrm{~K}$ and had a Lorentzian shape. We used the Gaussian half-width at half maximum HWHM=0.056 r.l.u. at $T=519.6 \mathrm{~K}$ for deconvoluting and determining the halfwidth at half maximum (HWHM) of the diffuse Lorentzian 

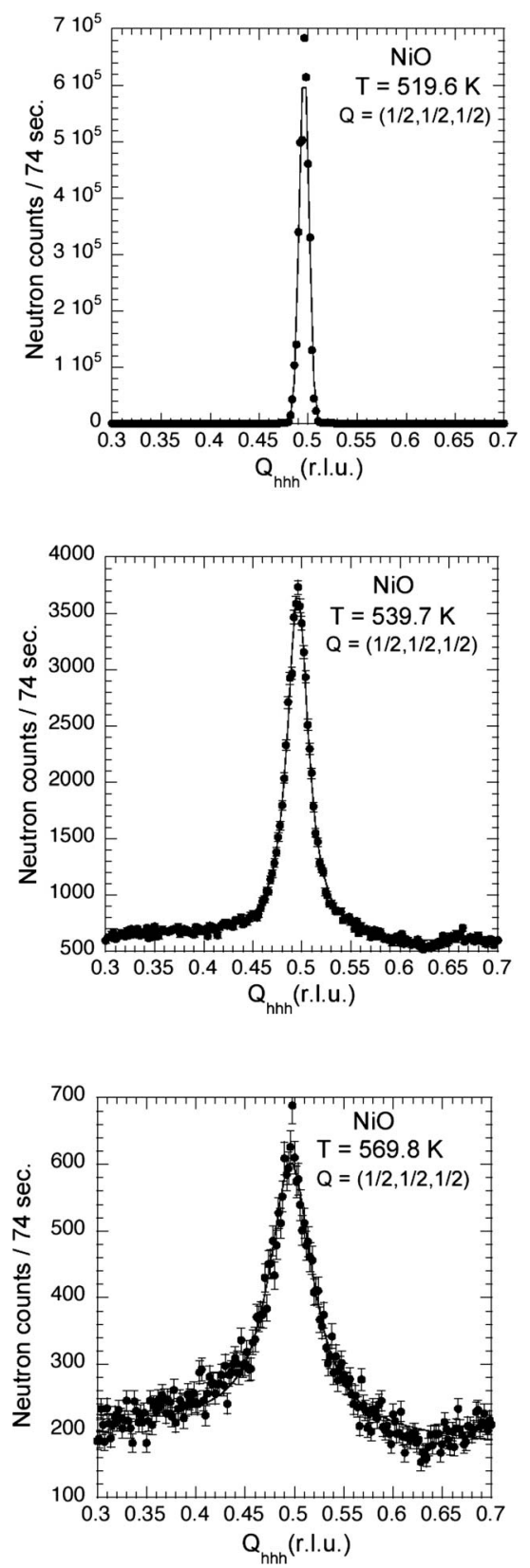

FIG. 2. $Q$ scans from $\mathrm{NiO}$ through $Q=\left(\frac{1}{2} \frac{1}{2} \frac{1}{2}\right)$ along [111] at temperatures below and above $T_{N}$. The continuous curves are results of fits with a Gaussian below $T_{N}$ and convoluted Lorentzian above $T_{N}$.

peak above $T_{N}$. The background on the right-hand side had a structure possibly due to the scattering from the sample environment materials. However this structure was far enough away from the peak and has not appreciably affected the HWHM and intensity derived from the fits. The Néel temperature $T_{N}$ and the critical exponent $\nu$ were determined by fitting the temperature dependence of the deconvoluted HWHM by the equation

$$
\mathrm{HWHM}=\left(\frac{T-T_{N}}{T_{N}}\right)^{\nu} .
$$

The least-squares fit gave $T_{N}=530 \pm 1 \mathrm{~K}$ and
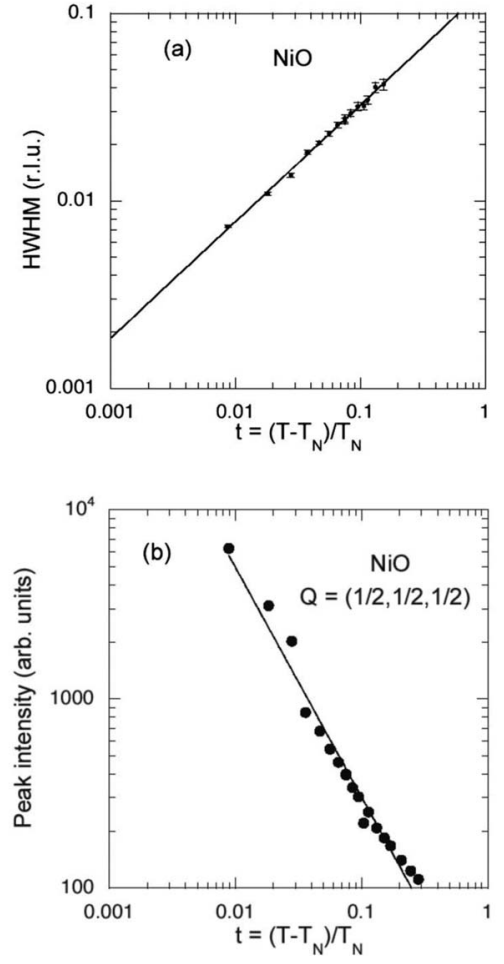

FIG. 3. (a) Log-log plot of the HWHM (inverse correlation length) vs reduced temperature. (b) Log-log plot of the intensity of the diffuse scattering (susceptibility) vs reduced temperature.

$\nu=0.64 \pm 0.03$. The fitted value of $T_{N}$ is the same within experimental accuracy as that determined from the temperature variation of the $\frac{1}{2} \frac{1}{2} \frac{1}{2}$ magnetic reflection. Figure $3(\mathrm{a})$ shows the log-log plot of the inverse correlation length or the HWHM vs reduced temperature. Figure 3(b) shows the log$\log$ plot of the peak intensity of the diffuse scattering vs reduced temperature. The slope of the resulting straight line gave the exponent $\gamma=1.22 \pm 0.04$. Although the log-log plot of the HWHM vs reduced temperature gave a straight line, the data points in the corresponding log-log plot for the diffuse intensity do not really lie on a straight line. This is due to the error in determining the weak diffuse scattering intensity with a high and structured background.

We can make the following remarks and conclusions from our experimental results:

(1) The antiferromagnetic phase transition in $\mathrm{NiO}$ is continuous within our experimental resolution. Experimentally we observed no discontinuity in the temperature variation in the intensity. Also $T_{N}$ determined from the data below $T_{N}$ is the same as that determined from above. This also indicates the phase transition is of the second order. These two $T_{N}$ determined from below and above should be different in the case of a first-order transition. We note that the critical exponent $^{23,24} \beta=0.328 \pm 0.002$ obtained by us is closer to three-dimensional Ising $(\beta=0.326)$ or three-dimensional $X Y$ value $(\beta=0.345)$ rather than the three-dimensional Heisenberg value $(\beta=0.367)$. In fact renormalization group theory ${ }^{25}$ and Monte Carlo simulations ${ }^{26-29}$ suggest a first-order transition for a $3 d$ type-II antiferromagnet. In a perfect fcc lattice, the order parameter has a dimensionality ${ }^{25}$ of $n=8$, 
where four dimensions come from the equivalence of the four $\langle 111\rangle$ ordering vectors, $\mathbf{k}$ - and the 2 from the spin components in the plane perpendicular to those. However, for several reasons, this model may not be relevant for bulk $\mathrm{NiO}$, as suggested by Mukamel and Krinsky. ${ }^{25}$ A fcc structure can be considered as consisting of four interpenetrating simple cubic sublattices. The coupling within a sublattice is given by the large $J_{2}$, causing a strong type-II antiferromagnetic ordering, with no frustration and a mean-field ordering temperature of about $4 S(S+1) J_{2}=884 \mathrm{~K}$ in the $\mathrm{NiO}$ case. However, in a mean-field sense, the sublattices do not interact no matter the value of $J_{1}$, because any given spin sees pairs of spins in opposite directions (not necessarily parallel to the given spin). Fluctuations ${ }^{25}$ may lift this degeneracy by creating a 4-k state, with $n=8$. Direct calculations ${ }^{31}$ and simulations ${ }^{32}$ for a spherical nanoparticle of $\mathrm{NiO}$ indeed find the 4-k ground state. However, the magnetically induced lattice distortion in bulk $\mathrm{NiO}$ lifts the degeneracy between the 12 nearest-neighbor interactions, yielding ${ }^{10} J_{1}^{-}=-16.1 \mathrm{~K}$ for interactions within the plane and $J_{1}^{+}=-15.7 \mathrm{~K}$ for interactions to neighboring planes. Hence, there is a small preference for ferromagnetic ordering within the plane, giving an interaction between the sublattices. Although the difference seems small, it removes the degeneracy (frustration) and in particular breaks the fourfold k-vector symmetry, leaving an $n=2$ system-or a $3 d X Y$ magnet, with $1-\mathbf{k}$ structure. NiO has predominantly a planar anisotropy, $D_{1}$, which contributes to confining the spins to the plane. It would surprising, if the very small in-plane anisotropy, $D_{2}$, is sufficient to induce an Ising character. However, with respect to the order, all the $3 d$ models, Ising $(n=1), X Y(n=2)$, and Heisenberg $(n=3)$ have continuous second-order transitions. Our present experimental results suggest a second-order continuous transition within experimental resolution. This is more complete and precise than previous measurements by van Doorn and DuPlessis, ${ }^{30}$ who also concluded the transition was continuous.

(2) The temperature dependence of the inverse correlation length (HWHM) has been fitted with a power-law critical exponent and the value of the exponent $\nu=0.64 \pm 0.03$ agrees more closely with the predicted value ${ }^{23,24}$ of $\nu=0.6312$ for an Ising system (dimensionality of the order parameter $n=1$, dimensionality of the system $d=3$ ) or $\nu=0.669$ for a $3 d X Y$ model $(n=1, d=3)$ than with the $\nu=0.707$ of a Heisenberg system $(n=3, d=3)$. Due to the uncertainty of the weak diffuse intensity data with high and structured background obtained during our present experiment we do not have much confidence on the determined $\gamma=1.22$. It is, however, closer to $3 d$ Ising (1.2378) or $3 d X Y$ value (1.316) than to the $3 d$ Heisenberg value 1.388. Also due to the lack of the critical exponent $\alpha$ for the specific heat we could not check the validity of Rushbrooke inequality. ${ }^{23,33}$

(3) The present investigation shows the presence of strong antiferromagnetic spin correlations well above the Néel temperature. Diffuse magnetic scattering of Lorentzian peak shape has been observed up to $T=800 \mathrm{~K} \approx 1.5 T_{N}$, the highest temperature at which the present measurements were performed. We however expect such strong spin correlations due to the inherent frustration of the fcc lattice that reduces the ordering temperature substantially relative to $T_{N}(\mathrm{MF})$ and gives rise to strong spin correlations above the ordering temperature.

In summary the present investigation shows that the AF phase transition in $\mathrm{NiO}$ is continuous within the experimental resolution. The critical exponents determined suggest that $\mathrm{NiO}$ should be regarded as a $3 d X Y$ system.
${ }^{1} \mathrm{P}$. Fulde, Electron Correlations in Molecules and Solids (Springer-Verlag, Berlin, 1991).

${ }^{2}$ P. Fulde, P. Thalmeier, and G. Zwicknagl, Solid State Physics (Elsevier, New York, 2006), Vol. 60, p. 1.

${ }^{3}$ Colossal Magnetoresistive Manganites, edited by T. Chatterji (Kluwer, Dordrecht, 2004).

${ }^{4}$ G. E. Bacon, Neutron Diffraction (Oxford University Press, Oxford, 1975).

${ }^{5}$ G. A. Sawatzky and J. W. Allen, Phys. Rev. Lett. 53, 2339 (1984).

${ }^{6}$ S. Hüfner, Adv. Phys. 43, 183 (1994).

${ }^{7}$ I. de P. R. Moreira, F. Illas, and R. L. Martin, Phys. Rev. B 65 , 155102 (2002).

${ }^{8}$ D. Ködderitzsch et al., Phys. Rev. B 66, 064434 (2002).

${ }^{9}$ W.-B. Zhang, Y.-L. Hu, K.-L. Han, and B.-Y. Tang, Phys. Rev. B 74, 054421 (2006).

${ }^{10}$ M. Hutchings and E. Samuelsen, Phys. Rev. B 6, 3447 (1972).

${ }^{11}$ T. Kotani and M. van Schilfgaarde, J. Phys.: Condens. Matter 20, 295214 (2008).

${ }^{12}$ P. W. Anderson, Phys. Rev. 115, 2 (1959).

${ }^{13}$ S. G. Chiuzbaian et al., Phys. Rev. Lett. 95, 197402 (2005).

${ }^{14}$ J. Kanamori, Prog. Theor. Phys. 17, 197 (1957).

${ }^{15}$ D. S. Rodbell and J. Owen, J. Appl. Phys. 35, 1002 (1964).

${ }^{16}$ S. Greenwald and J. S. Smart, Nature (London) 166, 523 (1950).

${ }^{17}$ Neutron Scattering from Magnetic Materials, edited by T. Chat- terji (Elsevier, Amsterdam, 2006).

${ }^{18}$ T. Chattopadhyay, T. Brückel, and P. Burlet, Phys. Rev. B 44, 7394 (1991).

${ }^{19}$ T. Chatterji et al., Phys. Rev. B 65, 134440 (2002).

${ }^{20}$ P.-A. Lindgard et al., J. Phys.: Condens. Matter 19, 286201 (2007).

${ }^{21}$ D. Hohlwein, Appl. Phys. A 74, S740 (2002).

${ }^{22}$ D. Hohlwein, J.-U. Hoffmann, and R. Schneider, Phys. Rev. B 68, 140408(R) (2003).

${ }^{23}$ M. F. Collins, Magnetic Critical Scattering (Oxford University Press, Oxford, 1989).

${ }^{24}$ P.-A. Lindgard, in Neutron Diffraction, edited by H. Dachs (Springer-Verlag, Berlin, 1978).

${ }^{25}$ D. Mukamel and S. Krinsky, Phys. Rev. B 13, 5065 (1976).

${ }^{26}$ K. Binder and D. P. Landau, Phys. Rev. B 30, 1477 (1984).

${ }^{27}$ T. M. Giebultowicz and J. K. Furdyna, J. Appl. Phys. 57, 3312 (1985).

${ }^{28}$ H. T. Diep and H. Kawamura, Phys. Rev. B 40, 7019 (1989).

${ }^{29}$ M. T. Heinilä and A. S. Oja, Phys. Rev. B 48, 16514 (1993).

${ }^{30}$ C. F. van Doorn and P. de V. Du Plessis, Phys. Lett. 66A, 141 (1978).

${ }^{31}$ R. H. Kodama and A. E. Berkowitz, Phys. Rev. B 59, 6321 (1999).

${ }^{32}$ P.-A. Lindgard, J. Magn. Magn. Mater. 266, 88 (2003).

${ }^{33}$ G. S. Rushbrooke, J. Chem. Phys. 39, 842 (1963). 